\title{
VARIATIONAL PRINCIPLES FOR GUIDED ELECTROMAGNETIC WAVES IN ANISOTROPIC MATERIALS
}

\author{
BY \\ WALTER HAUSER \\ Lincoln Laboratory, M.I.T., Lexington, Mass.
}

\begin{abstract}
It is the purpose of this paper to develop a general method for obtaining approximate solutions to the problems of the propagation of waves in a guide which may only be partially filled with material having tensor electromagnetic properties. With the introduction of an appropriate dyadic Green's function we are able to obtain a formal solution to the problem in terms of an integral involving the field vectors in the perturbing rod. The reformulation of the original problem in terms of an integral equation enables us to construct a variational principle whose extremal value is insensitive to a great range of trial functions. The extremal value of the variational expression from which the integral equations for the field are derivable is shown to be proportional to $\left(\gamma_{0}^{2}-\gamma^{2}\right)$, the difference between the square of the propagation constant of the wave in the empty and loaded guide. Normal modes in terms of which an expansion of the dyadic Green's function is obtained are defined. In the last section we demonstrate the ability of the variational method to improve the results obtained by perturbation methods obtaining a first order approximation for the propagation constant of a wave in a rectangular guide containing an infinite ferrite slab.
\end{abstract}

Introduction. With the use of ferrites in the design of microwave gyrators, circulators and nonreciprocal transmission systems, one has become interested in the problem of cavities and waveguides containing materials having tensor electromagnetic properties. In general, such a problem is extremely difficult and tedious. One is almost impelled to look for approximate solutions. In those problems where the material fills but a small part of the cavity or guide, or where the electromagnetic properties of the perturbing material differ but slightly from those of the empty guide or cavity, perturbation theory has been somewhat successful in obtaining first order approximations [1]. There exist a number of problems, however, to which perturbation theory yields poor or no results.

This paper is the first of a group of papers in which we shall concern ourselves with the development and application of a general method for obtaining approximate solutions to the problems of waveguides and cavities containing materials with tensor electromagnetic properties. The method is an extension of the work of Schwinger [2] on the problem of isotropic obstacles in cavities. With the introduction of an appropriate dyadic Green's function we are able to obtain a formal solution to the problem in terms of integrals involving the field vectors in the perturbing material. While the resulting integral equations are not any easier to solve, there exists the advantage of being able to construct stationary expressions for the quantities of interest from which the integral equations for the fields within the material are derivable. Consequently, we have a very powerful method for obtaining approximate solutions for these quantities. In this paper, for example, we concern ourselves with the problem of the propagation of waves in a guide which may only be partially filled with a rod of uniform cross-section.

Received July 26, 1957. The research reported in this document was supported jointly by the Army, Navy and Air Force under contract with Massachusetts Institute of Technology. 
In this case, the first order variation of $\left(\gamma_{0}^{2}-\gamma^{3}\right)$, the difference between the square of the propagation constant of the wave in the empty and loaded guide, is zero with respect to similar variations of the fields in the rod. Berk [3] has treated these problems obtaining variational principles which yield the differential equations satisfied by the electromagnetic field. His expressions, however, are not applicable to lossy media in general, and furthermore require a knowledge of the fields everywhere within the guide. Our method in addition provides us with a systematic way of improving the trial wave function, thus permitting us to improve upon the results obtained by perturbation formulae.

While the full use of the variational method consists of the substitution of trial functions with unknown variational parameters followed by the calculation of the stationary quantity, we may at times obtain good first order results by simply substituting completely determined trial functions. In such cases the variational expression reduces essentially to a perturbation formula [4]. It has the advantage, however, of furnishing the best amplitude of the trial field within the perturbing rod.

I. Integral equations for the electromagnetic field. The problem of a waveguide partially or completely filled by a rod of uniform cross-section permits separation with respect to the coordinate of the guide axis which we choose as the $z$-axis. Assuming an $\exp (j \omega t)$ time dependence, we can write the electric and magnetic fields as

$$
\mathbf{E}(x, y) \exp [j(\omega t+\gamma z)]
$$

and

$$
\mathbf{H}(x, y) \exp [j(\omega t+\gamma z)]
$$

respectively, where $\gamma$, the propagation constant, is in general a complex quantity.

In terms of $\mathbf{E}(x, y)$ and $\mathbf{H}(x, y)$, the $z$ independent parts of the electromagnetic field, Maxwell's equations in a region free of charges and currents reduce to

$$
\begin{aligned}
& \left(\nabla_{t}+j \gamma \mathbf{k}\right) \times \mathbf{E}=-j \omega \boldsymbol{u} \cdot \mathbf{H}, \\
& \left(\nabla_{t}+j \gamma \mathbf{k}\right) \times \mathbf{H}=j \omega \mathbf{\varepsilon} \cdot \mathbf{E} .
\end{aligned}
$$

$\varepsilon$ is the tensor electric permittivity, and $\boldsymbol{u}$ is the tensor magnetic permeability of the medium filling the guide. In the usual manner, by taking the curl of Maxwell's equations one obtains the wave equations satisfied by $\mathbf{E}$ and $\mathbf{H}$. We find

$$
\mathbf{d} \times \mathbf{d} \times \mathbf{E}-\omega^{2} \epsilon_{0} \mu_{0} \mathbf{E}=\omega^{2} \mu_{0}\left(\boldsymbol{z}^{\prime} \cdot \mathbf{E}\right)-j \omega \mathbf{d} \times\left(\boldsymbol{u}^{\prime} \cdot \mathbf{H}\right),
$$

and

$$
\mathbf{d} \times \mathbf{d} \times \mathbf{H}-\omega^{2} \epsilon_{0} \mu_{0} \mathbf{H}=\omega^{2} \epsilon_{0}\left(\boldsymbol{u}^{\prime} \cdot \mathbf{H}\right)+j \omega \mathbf{d} \times\left(\varepsilon^{\prime} \cdot \mathbf{E}\right) .
$$

We have set

$$
\begin{aligned}
\mathbf{d} & =\nabla_{\mathfrak{t}}+j \boldsymbol{\gamma} \mathbf{k}, \\
\boldsymbol{\varepsilon} & =\boldsymbol{\epsilon}_{0} \mathbf{I}+\boldsymbol{\varepsilon}^{\prime}, \\
\boldsymbol{u} & =\mu_{0} \mathbf{I}+\boldsymbol{u}^{\prime},
\end{aligned}
$$

where $I$ is the unit dyadic, and $\epsilon_{0}$ and $\mu_{0}$ are scalars whose choice depends on the problem at hand.

From the form of Maxwell's equations, we realize that the boundary conditions on 
E, $\mathbf{H}, \mathbf{B}$, and $\mathbf{D}$ are not affected by the introduction of tensor $\varepsilon$ and $\boldsymbol{u}$. Across a surface where $\varepsilon$ or $\boldsymbol{u}$ or both change discontinuously, the tangential components of $\mathbf{E}$ and $\mathbf{H}$, and the normal components of $\mathbf{B}$ and $\mathbf{D}$ are continuous. At a conducting surface we shall require the tangential component of $\mathbf{E}$ and the normal component of $\mathbf{B}$ to be zero.

At this point we should also like to introduce the adjoint.fields, $\mathbf{E}^{a}$ and $\mathbf{H}^{a}$, which we take as the solutions of the equations

$$
\begin{aligned}
\mathbf{d}^{a} \times \mathbf{E}^{a} & =j \omega \mathbf{H}^{a} \cdot \boldsymbol{u}=j \omega \mathbf{B}^{a}, \\
\mathbf{d}^{a} \times \mathbf{H}^{a} & =-j \omega \mathbf{E}^{a} \cdot \varepsilon=-j \omega \mathbf{D}^{a},
\end{aligned}
$$

where $\mathrm{d}^{a}=\nabla_{\imath}-j \gamma \mathbf{k}$.

In the case of lossless media where $\varepsilon$ and $\boldsymbol{u}$ are hermitian [5], $\mathbf{E}^{a}=\mathbf{E}^{*}$ and $\mathbf{H}^{a}=\mathbf{H}^{*}$. In the case of lossy media, the adjoint fields may also be simply related to the fields $\mathbf{E}$ and $\mathbf{H}$. For example in the case of lossy ferrites, taking Lax's form of the magnetic permeability tensor [4], having its diagonal elements even in $\omega$ and its off diagonal elements odd in $\omega$,

and

$$
\mathbf{H}^{a}(x, y, \gamma, \omega)=\mathbf{H}(x, y,-\gamma,-\omega),
$$

$$
\mathbf{E}^{a}(x, y, \gamma, \omega)=\mathbf{E}(x, y,-\gamma,-\omega) .
$$

Consider now the problem of a waveguide of cross-section $\left(S_{1}+S_{2}\right)$ with region $S_{1}$ occupied by a material having tensor electromagnetic properties. We are confronted with the problem of solving Eqs. (1) within the two regions subject to the boundary conditions stated above. To do so, we* employ the help of the dyadic Green's function which satisfies the differential equation

$$
\mathbf{d}^{a} \times \mathbf{d}^{a} \times \mathbf{Z}^{a}\left(\mathbf{x} \mid \mathbf{x}^{\prime}\right)-\omega^{2} \epsilon_{0} \mu_{0} Z^{a}\left(\mathbf{x} \mid \mathbf{x}^{\prime}\right)=\mathbf{I} \delta\left(\mathbf{x}-\mathbf{x}^{\prime}\right)
$$

everywhere in the guide, where $\mathbf{x}=x \mathbf{i}+y \mathbf{j}$.

The Dirac delta function $\delta\left(\mathbf{x}-\mathbf{x}^{\prime}\right)=\delta\left(x-x^{\prime}\right) \delta\left(y-y^{\prime}\right)$. We further require $\mathbf{Z}^{\mathbf{a}}$ to satisfy certain boundary conditions at the boundary of the guide. The Green's function $\mathbf{N}^{a}\left(\mathbf{x} \mid \mathbf{x}^{\prime}\right)$, which satisfies the condition

$$
\mathbf{n} \times\left[\mathbf{N}^{a}\left(\mathbf{x} \mid \mathbf{x}^{\prime}\right)\right]=\mathbf{0}
$$

when $\mathbf{x}$ lies on the boundary of the guide, we refer to as the electric dyadic Green's function.

Analogously the dyadic Green's function, $\mathbf{M}^{a}\left(\mathbf{x} \mid \mathbf{x}^{\prime}\right)$, which satisfies the condition

$$
\mathbf{n} \times\left[\mathbf{d}^{a} \times \mathbf{M}^{a}\left(\mathbf{x} \mid \mathbf{x}^{\prime}\right)\right]=0,
$$

when $x$ lies on the boundary of the guide, we call the magnetic dyadic Green's function.

For the solution of the fields in problems where either $\mathbf{E}$ or $\mathbf{H}$ is divergenceless, we further require the respective dyadic Green's function to satisfy the condition

$$
\mathbf{d}^{a} \cdot \mathbf{Z}^{a}\left(\mathbf{x} \mid \mathbf{x}^{\prime}\right)=0 .
$$

We now rewrite Eq. (3) in the form

$$
\nabla_{\imath} \times\left(\mathbf{d}^{a} \times \mathbf{N}_{\alpha}^{a}\right)-j \gamma \mathbf{k} \times\left(\mathbf{d}^{a} \times \mathbf{N}_{\alpha}^{a}\right)-k_{0}^{2} \mathbf{N}_{\alpha}^{a}=\boldsymbol{\alpha} \delta\left(\mathbf{x}-\mathbf{x}^{\prime}\right),
$$

where we have set $k_{0}^{2}=\omega^{2} \epsilon_{0} \mu_{0}$ and $\mathbf{N}_{\alpha}^{a}=\mathbf{N}^{a} \cdot \boldsymbol{\alpha}$. $\boldsymbol{\alpha}$ is an arbitrary vector introduced in

*We follow closely the work of J. Schwinger [2] on the problem of obstacles in cavities. 
order to simplify the handling of the dyadic Green's function. Taking the dot product of Eq. (2a) with $\mathbf{N}_{\alpha}^{a}$, the above equation with $\mathbf{E}$ and subtracting, we find that

$$
\begin{aligned}
\mathbf{E} \cdot \boldsymbol{\alpha} \delta\left(\mathbf{x}-\mathbf{x}^{\prime}\right)=\nabla_{\imath} \cdot\left[\mathbf{N}_{\alpha}^{a} \times(\mathbf{d} \times \mathbf{E})-\mathbf{E} \times\left(\mathbf{d}^{a} \times \mathbf{N}_{\alpha}^{a}\right)+j \omega \mathbf{N}_{\alpha}^{a} \times\left(\boldsymbol{u}^{\prime} \cdot \mathbf{H}\right)\right] \\
+\omega^{2} \mu_{0}\left(\mathbf{\varepsilon}^{\prime} \cdot \mathbf{E}\right) \cdot \mathbf{N}_{\alpha}^{a}-j \omega\left(\mathbf{d}^{a} \times \mathbf{N}_{\alpha}^{a}\right) \cdot\left(\boldsymbol{u}^{\prime} \cdot \mathbf{H}\right) .
\end{aligned}
$$

Utilization of Maxwell's equations and the boundary conditions satisfied by the tangential components of $\mathbf{E}$ and $\mathbf{H}$, leads to*

$$
\begin{gathered}
\mathbf{E}\left(\mathbf{x}^{\prime}\right) \cdot \boldsymbol{\alpha}=\omega^{2} \mu_{0} \int \mathbf{N}_{\alpha}^{a}\left(\mathbf{x} \mid \mathbf{x}^{\prime}\right) \cdot \mathbf{\varepsilon}^{\prime} \cdot \mathbf{E}(\mathbf{x}) d S-j \omega \int\left[\mathbf{d}^{a} \times \mathbf{N}_{\alpha}^{a}\left(\mathbf{x} \mid \mathbf{x}^{\prime}\right)\right] \cdot\left[\mathbf{u}^{\prime} \cdot \mathbf{H}(\mathbf{x})\right] d S . \\
\int \nabla_{\boldsymbol{t}} \cdot \mathbf{P}(x, y) d x d y=\Phi_{\mathbf{n}} \cdot \mathbf{P} d s .
\end{gathered}
$$

The magnetic field may be computed directly from the previous equation through the use of Eq. (1), or it may be obtained by utilizing Eq. (2b) and the magnetic dyadic Green's function. By repeating the steps which led to Eq. (4), we obtain

$$
\mathbf{H}\left(\mathbf{x}^{\prime}\right) \cdot \boldsymbol{\alpha}=\omega^{2} \epsilon_{0} \int \mathbf{M}_{\alpha}^{a} \cdot\left(\mathbf{u}^{\prime} \cdot \mathbf{H}\right) d S+j \omega \int\left(\mathbf{d}^{a} \times \mathbf{M}_{\alpha}^{a}\right) \cdot\left(\boldsymbol{\varepsilon}^{\prime} \cdot \mathbf{E}\right) d S .
$$

With the help of the reciprocity relations satisfied by the Green's functions it is readily shown that the two methods for obtaining $\mathbf{H}$ are equivalent [6].

Utilizing the two dimensional Green's second identity

$$
\begin{aligned}
\int\left[\mathbf{A}^{a} \cdot(\mathbf{d} \times \mathbf{d} \times \mathbf{B})-\mathbf{B} \cdot\left(\mathbf{d}^{a} \times \mathbf{d}^{a} \times \mathbf{A}^{a}\right)\right] d S & \\
& =\oint \mathbf{n} \cdot\left[\mathbf{B} \times\left(\mathbf{d}^{a} \times \mathbf{A}^{a}\right)-\mathbf{A}^{a} \times(\mathbf{d} \times \mathbf{B})\right] d s,
\end{aligned}
$$

we find upon setting

$$
\mathbf{A}^{a}=\mathbf{Z}_{\alpha}^{a}\left(\mathbf{x} \mid \mathbf{x}^{\prime}\right)
$$

and

$$
\mathbf{B}=\mathbf{Z}_{\beta}\left(\mathbf{x} \mid \mathbf{x}^{\prime \prime}\right),
$$

that

$$
\mathbf{Z}\left(\mathbf{x}^{\prime \prime} \mid \mathbf{x}^{\prime}\right)=\left[\mathbf{Z}^{a}\left(\mathbf{x}^{\prime} \mid \mathbf{x}^{\prime \prime}\right)\right]^{T},
$$

where $\mathbf{Z}^{+}$is the transpose of $\mathbf{Z}$. Similarly, setting

$$
\mathbf{A}^{a}=\mathbf{d}^{a} \times \mathbf{M}_{\alpha}^{a}\left(\mathbf{x} \mid \mathbf{x}^{\prime}\right)
$$

and

$$
\mathbf{B}=\mathbf{N}_{\beta}\left(\mathbf{x} \mid \mathbf{x}^{\prime \prime}\right),
$$

we obtain

$$
\mathbf{d}^{a} \times \mathbf{M}^{a}\left(\mathbf{x}^{\prime \prime} \mid \mathbf{x}^{\prime}\right)=\left[\mathbf{d} \times \mathbf{N}\left(\mathbf{x}^{\prime} \mid \mathbf{x}^{\prime \prime}\right)\right]^{T} .
$$

II. The normal mode expansion of the dyadic Green's function. Our next task is

*We have used the two dimensional Green's theorem 
that of finding an explicit expression for the dyadic Green's functions. Such an expression can, of course, take the form of an expansion in terms of the complete set of vector eigenfunctions satisfying the homogeneous equation

$$
\mathbf{d}^{a} \times \mathbf{d}^{a} \times \mathbf{A}_{n}^{a}-\left(\kappa_{n}^{2}+\gamma^{2}\right) \mathbf{A}_{n}^{a}=0,
$$

the boundary condition

$$
\mathbf{n} \times \mathbf{A}_{n}^{a}=\mathbf{0}
$$

for the electric modes, and the boundary condition

$$
\mathbf{n} \times\left(\mathbf{d}^{a} \times \mathbf{A}_{n}^{a}\right)=0
$$

for the magnetic modes.

We can readily find such a set of vector eigenfunctions in terms of the usual normal modes of the guide. If $E_{z n}$ and $H_{z n}$ are the $z$ independent parts of the $z$ components of the usual TM and TE modes of the guide, we find that the vectors

$$
\mathbf{F}_{n}^{a}\left(x, y, \gamma, \gamma_{n}, \omega\right)=\left\{\begin{array}{l}
\frac{j \omega \mu_{0}}{\gamma_{n}^{2}-\omega^{2} \epsilon_{0} \mu_{0}} \mathbf{k} \times \nabla_{t} H_{z n}^{a} \\
{\left[\frac{-j \gamma_{n}}{\gamma_{n}^{2}-\omega^{2} \epsilon_{0} \mu_{0}} \nabla_{t}-\frac{\gamma_{n}}{\gamma} \mathbf{k}\right] E_{z n}^{a},}
\end{array}\right.
$$

where

$$
E_{z n}^{a}(x, y)=E_{2 n}^{*}(x, y), \quad H_{z n}^{a}(x, y)=H_{2 n}^{*}(x, y)
$$

and

$$
\mathbf{F}_{n}\left(x, y, \gamma, \gamma_{n}, \omega\right)=\left\{\begin{array}{l}
\frac{-j \omega \mu_{0}}{\gamma_{n}^{2}-\omega^{2} \epsilon_{0} \mu_{0}} \mathbf{k} \times \nabla_{t} H_{z n} \\
{\left[\frac{j \gamma_{n}}{\gamma_{n}^{2}-\omega^{2} \epsilon_{0} \mu_{0}} \nabla_{t}-\frac{\gamma_{n}}{\gamma} \mathbf{k}\right] E_{z n}}
\end{array}\right.
$$

satisfy Eq. (6) and the boundary conditions which have to be satisfied by the electric modes. In addition they are divergenceless,

$$
\mathbf{d}^{a} \cdot \mathbf{F}_{n}^{a}=0 .
$$

For completeness, we need another set of vector eigenfunctions whose curl is zero,

$$
\mathbf{d}^{a} \times \mathbf{f}_{n}^{a}=0 .
$$

Such a function, which is the gradient of a scalar,

$$
\mathbf{f}_{n}^{a}=\mathbf{d}^{a} \phi_{n}^{a}
$$

will satisfy Eq. (6) for

$$
\kappa_{n}^{2}=-\gamma^{2},
$$

and the boundary condition $\mathbf{n} \times \mathbf{f}_{n}^{a}=0$, if $\phi_{n}^{a}=0$ on the boundary. In order however, for the functions $f_{n}^{a}$ to form an orthogonal set among themselves, (they are automatically orthogonal to the functions $\mathbf{F}_{n}$ ), we choose for the set of scalar functions $\phi_{n}^{a}$, the set satisfying the equation

$$
\left(\mathbf{d}^{a} \cdot \mathbf{d}^{a}+\omega_{n}^{2}\right) \phi_{n}^{a}=0 .
$$


Analogously we obtain the magnetic vector eigenfunctions, the divergenceless vector eigenfunctions,

$$
\mathbf{G}_{n}^{a}\left(x, y, z, \gamma, \gamma_{n}, \omega\right)=\left\{\begin{array}{l}
{\left[\frac{-j \gamma_{n}}{\gamma_{n}^{2}-\omega^{2} \epsilon_{0} \mu_{0}} \nabla_{\imath}-\frac{\gamma_{n}}{\gamma} \mathbf{k}\right] H_{s n}^{a},} \\
\frac{-j \omega \epsilon_{0}}{\gamma_{n}^{2}-\omega^{2} \epsilon_{0} \mu_{0}} \mathbf{k} \times \nabla_{\imath} E_{s n}^{a},
\end{array}\right.
$$

and the curl-less eigenfunctions,

$$
\mathbf{g}_{n}^{a}=\mathbf{d}^{a} \psi_{n}^{a},
$$

with $\mathbf{n} \cdot \mathbf{d}^{2} \psi_{n}^{a}=0$ on the boundary. The functions $g_{n}^{a}$ are orthogonal to each other and the functions $G_{n}$ if the scalar functions $\psi_{n}^{a}$ satisfy the equation

$$
\left(d^{a} \cdot d^{a}+\omega_{n}^{\prime 2}\right) \psi_{n}^{a}=0 .
$$

We now demonstrate the orthogonality of the vector eigenfunctions as expressed by

$$
\begin{aligned}
\int \mathbf{F}_{n} \cdot \mathbf{F}_{m}^{a} d S & =\Lambda_{n}^{2} \delta_{n m}, \\
\int \mathbf{f}_{n} \cdot \mathbf{f}_{m}^{a} d S & =\Omega_{n}^{2} \delta_{n m}, \\
\int \mathbf{f}_{n} \cdot \mathbf{F}_{m}^{a} d S & =0, \\
\int \mathbf{G}_{n} \cdot \mathbf{G}_{m}^{a} d S & =\lambda_{n}^{2} \delta_{n m}, \\
\int \mathbf{g}_{n} \cdot \mathbf{g}_{m}^{a} d S & =\eta_{n}^{2} \delta_{n m}, \\
\int \mathbf{G}_{n} \cdot \mathbf{g}_{m}^{a} d S & =0 .
\end{aligned}
$$

Equations (a), (c), (d) and (f) are readily obtainable through the use of Eq. (6), whose right hand side vanishes if $\mathbf{A}$ and $\mathbf{B}$ are both either electric or magnetic vector eigenfunctions. From Eq. (7) it then follows that

$$
\left(\kappa_{n}^{2}-\kappa_{m}^{2}\right) \int \mathbf{A}_{n}^{a} \cdot \mathbf{B}_{m} d S=0 .
$$

Equations (b) and (e) can be shown to hold since for $\theta_{n}$ being any scalar function

$$
\begin{aligned}
\int\left(\mathbf{d} \theta_{m}\right) \cdot\left(\mathbf{d}^{a} \theta_{n}^{a}\right) d S=\int \nabla_{t} \cdot\left(\theta_{m} \mathbf{d}^{a} \theta_{n}^{a}\right) d S-\int \theta_{m}\left(\mathbf{d}^{a} \cdot \mathbf{d}^{a} \theta_{n}^{a}\right) d S & \\
& =\int \nabla_{t} \cdot\left[\left(\mathbf{d} \theta_{m}\right) \theta_{n}^{a}\right] d S-\int \theta_{n}^{a} \mathbf{d} \cdot \mathbf{d} \theta_{m} d S .
\end{aligned}
$$

If now $\theta_{n}$ satisfies the equation

$$
\mathrm{d}^{a} \cdot \mathrm{d}^{a} \theta_{n}^{a}+\omega_{n}^{2} \theta_{n}^{a}=0
$$


and the boundary condition satisfied either by $\phi_{n}$ or $\psi_{n}$, we have that

$$
\begin{aligned}
\int \mathrm{d} \theta_{m} \cdot \mathrm{d}^{a} \theta_{n} d S & =\omega_{n}^{2} \int \theta_{m} \theta_{n}^{a} d S \\
& =\omega_{m}^{2} \int \theta_{m} \theta_{n}^{a} d S .
\end{aligned}
$$

Thus if $\omega_{n}^{2} \neq \omega_{m}^{2}$ the left hand side must be zero. We assume that degenerate eigenfunctions have been orthogonalized. It is readily shown that the divergenceless electric and magnetic vector eigenfunctions are related through the equations

$$
\mathbf{d} \times \mathbf{F}_{n}=-j \omega \mu_{0} \mathbf{G}_{n} \alpha_{n}
$$

and

$$
\mathbf{d} \times \mathrm{G}_{n}=j \omega \epsilon_{0} \mathbf{F}_{n} \beta_{n}
$$

where

$$
\alpha_{n}=\left\{\begin{array}{cc}
\frac{\gamma}{\gamma_{n}} & \text { for TE modes } \\
\frac{\gamma_{n}}{\gamma} \frac{k_{0}^{2}+\gamma^{2}-\gamma_{n}^{2}}{k_{0}^{2}} & \text { for TM modes }
\end{array}\right.
$$

and

$$
\beta_{n}=\left\{\begin{array}{cl}
\frac{\gamma_{n}}{\gamma} \frac{k_{0}^{2}+\gamma^{2}-\gamma_{n}^{2}}{k_{0}^{2}} & \text { for TE modes } \\
\frac{\gamma}{\gamma_{n}} & \text { for TM modes }
\end{array}\right.
$$

The functions $F_{n}$ and $f_{n}$ together form a complete set of orthogonal vector eigenfunctions which may be used to represent any vector field having zero tangential component on the boundary of the guide. Similarly the set of orthogonal vector eigenfunctions $\mathbf{G}_{n}$ and $g_{n}$ may be used to represent any vector field satisfying the same boundary conditions as those imposed upon the magnetic vector eigenfunctions. We should thus be able to write

$$
\mathbf{N}_{\alpha}^{a}\left(\mathbf{x} \mid \mathbf{x}^{\prime}\right)=\sum_{n} \mathfrak{a}_{n} \mathbf{F}_{n}^{a}+\sum_{n} \mathfrak{b}_{n} \mathfrak{f}_{n}^{a}
$$

and

$$
\mathbf{M}_{\alpha}^{a}\left(\mathbf{x} \mid \mathbf{x}^{\prime}\right)=\sum_{n} \mathfrak{a}_{n}^{\prime} \mathbf{G}_{n}^{a}+\sum_{n} \mathfrak{b}_{n}^{\prime} \mathfrak{g}_{n}^{a} .
$$

Realizing that the normal modes satisfy Eq. (2) with $\varepsilon^{\prime}$ and $\boldsymbol{u}^{\prime}$ equal to zero and $k_{0}^{2}=\omega^{2} \epsilon_{0} \mu_{0}$ replaced by $\left(\kappa_{n}^{2}+\gamma^{2}\right)$, we find

$$
\mathbf{A}_{n}\left(\mathbf{x}^{\prime}\right) \cdot \boldsymbol{\alpha}=\left(\kappa_{n}^{2}+\gamma^{2}-k_{0}^{2}\right) \int \mathbf{A}_{n}(\mathbf{z}) \cdot \mathbf{Z}_{\alpha}^{a}\left(\mathbf{x} \mid \mathbf{x}^{\prime}\right) d S
$$

as a solution of Eq. (7). Inserting expansions (12) and (13) for the dyadic Green's functions into this integral equation, and utilizing the orthogonality relations (10), we find 
that

$$
\begin{aligned}
& \mathfrak{a}_{n}=\frac{\mathbf{F}_{n}\left(\mathbf{x}^{\prime}\right) \cdot \boldsymbol{\alpha}}{\left(\gamma^{2}-\gamma_{n}^{2}\right) \Lambda_{n}^{2}}, \\
& \mathfrak{b}_{n}=\frac{-\mathbf{f}_{n}\left(\mathbf{x}^{\prime}\right) \cdot \boldsymbol{\alpha}}{k_{0}^{2} \Omega_{n}^{2}}, \\
& \mathfrak{a}_{n}^{\prime}=\frac{\mathbf{G}_{n}\left(\mathbf{x}^{\prime}\right) \cdot \boldsymbol{\alpha}}{\left(\gamma^{2}-\gamma_{n}^{2}\right) \lambda_{n}^{2}},
\end{aligned}
$$

and

$$
\mathfrak{b}_{n}^{\prime}=-\frac{\mathbf{g}_{n}\left(\mathbf{x}^{\prime}\right) \cdot \boldsymbol{\alpha}}{k_{0}^{2} \eta_{n}^{2}} .
$$

We have thus found the expansion of the dyadic Green's function in terms of the normal modes,

$$
\mathbf{N}^{a}\left(\mathbf{x} \mid \mathbf{x}^{\prime}\right)=\sum_{n} \frac{\mathbf{F}_{n}^{a}(\mathbf{x}) \mathbf{F}_{n}\left(\mathbf{x}^{\prime}\right)}{\left(\gamma^{2}-\gamma_{n}^{2}\right) \Lambda_{n}^{2}}-\frac{1}{k_{0}^{2}} \sum_{n} \frac{\mathbf{f}_{n}^{a}(\mathbf{x}) \mathbf{f}_{n}\left(\mathbf{x}^{\prime}\right)}{\Omega_{n}^{2}}
$$

and

$$
\mathbf{M}^{a}\left(\mathbf{x} \mid \mathbf{x}^{\prime}\right)=\sum_{n} \frac{\mathbf{G}_{n}^{a}(\mathbf{x}) \mathbf{G}_{n}\left(\mathbf{x}^{\prime}\right)}{\left(\gamma^{2}-\gamma_{n}^{2}\right) \lambda_{n}^{2}}-\frac{1}{k_{0}^{2}} \sum_{n} \frac{\mathbf{g}_{n}^{a}(\mathbf{x}) \mathbf{g}_{n}\left(\mathbf{x}^{\prime}\right)}{\eta_{n}^{2}}
$$

For those problems where $\mathbf{N}$ or $\mathbf{M}$ is taken to be divergenceless, the sum over the $\mathbf{f}_{n}$ 's or $\mathbf{g}_{n}$ 's respectively, except for the terms which are also divergenceless, are not to be included.

III. Variational principles. In terms of the normal mode expansion of the dyadic Green's function

$$
\mathbf{E}\left(\mathbf{x}^{\prime}\right)=\sum_{n=0}^{\infty} \frac{J_{n}^{e} \mathbf{E}_{n}\left(\mathbf{x}^{\prime}\right)}{\left(\gamma^{2}-\gamma_{n}^{2}\right)}-\frac{\omega^{2} \mu_{0}}{k_{0}^{2}} \sum_{n=0}^{\infty} \frac{\left[\int \mathbf{f}_{n}^{a} \cdot \mathbf{\varepsilon}^{\prime} \cdot \mathbf{E} d S\right] \mathbf{f}_{n}\left(\mathbf{x}^{\prime}\right)}{\Omega_{n}^{2}},
$$

where having set

$$
\mathbf{E}_{n}=\left(\beta_{n}\right)^{1 / 2} \mathbf{F}_{n}
$$

and

$$
\begin{gathered}
\mathbf{H}_{n}=\left(\alpha_{n}\right)^{1 / 2} \mathbf{G}_{n}, \\
J_{n}^{e}=\frac{\omega^{2} \mu_{0}}{\beta_{n} \Lambda_{n}^{2}}\left[\int \mathbf{E}_{n}^{a} \cdot \mathbf{\varepsilon}^{\prime} \cdot \mathbf{E} d S+\left(\alpha_{n} \beta_{n}\right)^{1 / 2} \int \mathbf{H}_{n}^{a} \cdot \mathbf{u}^{\prime} \cdot \mathbf{H} d S\right] .
\end{gathered}
$$

Taking the curl of Eq. (16) yields

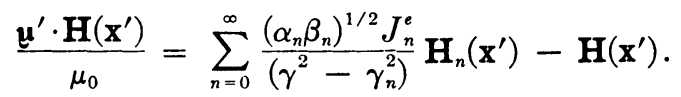

We note again, that in those problems where $\mathbf{E}$ is divergenceless we shall omit the last sum in Eq. (16), except for the $\mathbf{f}_{0}$ term which is also divergenceless. 
Analogous to the foregoing we find

$$
\mathbf{E}^{a}(\mathbf{x})=\sum_{n=0}^{\infty} \frac{\left(J_{n}^{e}\right)^{a} \mathbf{E}_{n}^{a}(\mathbf{x})}{\left(\gamma^{2}-\gamma_{0}^{2}\right)}-\frac{\omega^{2} \mu_{0}}{k_{0}^{2}} \sum_{n=0}^{\infty} \frac{\int \mathbf{E}^{a} \cdot \mathbf{\varepsilon}^{\prime} \cdot \mathbf{f}_{n} d S}{\Omega_{0}^{2}} \mathbf{f}_{n}^{a}(\mathbf{x})
$$

and

$$
\frac{\mathbf{H}^{a}(\mathbf{x}) \cdot \boldsymbol{u}^{\prime}}{\boldsymbol{\mu}_{0}}=\sum_{n=0}^{\infty} \frac{\left(\alpha_{n} \beta_{n}\right)^{1 / 2}\left(J_{n}^{e}\right)^{a} \mathbf{H}_{n}^{a}(\mathbf{x})}{\left(\gamma^{2}-\gamma_{0}^{2}\right)}-\mathbf{H}^{a}(\mathbf{x}) .
$$

From the wave equations satisfied by the electromagnetic field and the normal modes, we obtain

$$
\begin{aligned}
\nabla_{\imath} \cdot\left[\mathbf{E} \times \mathbf{d}^{a} \times \mathbf{E}_{n}^{a}+(\mathbf{d} \times \mathbf{E}) \times \mathbf{E}_{n}^{a}-j \omega \mathbf{E}_{n}^{a} \times\left(\boldsymbol{u}^{\prime} \cdot \mathbf{H}\right)\right]+\left(\gamma^{2}-\gamma_{n}^{2}\right) \mathbf{E}_{n}^{a} \cdot \mathbf{E} \\
=\omega^{2} \mu_{0} \mathbf{E}_{n}^{a} \cdot \mathbf{\varepsilon}^{\prime} \cdot \mathbf{E}-j \omega\left(\mathbf{d}^{a} \times \mathbf{E}_{n}^{a}\right) \cdot\left(\boldsymbol{u}^{\prime} \cdot \mathbf{H}\right) .
\end{aligned}
$$

It follows therefore that

$$
J_{n}^{e}=\left(\gamma^{2}-\gamma_{n}^{2}\right) \int \mathbf{E}_{n}^{a} \cdot \mathbf{E} d S / \beta_{n} \Lambda_{n}^{2} .
$$

We can fix the amplitude of the field within the rod by restricting ourselves to fields satisfying the condition $\int \mathbf{E}_{0}^{a} \cdot \mathbf{E} d S=\left(\beta_{0}\right)^{1 / 2} \Lambda_{0}^{2}$, where $E_{0}$ is the unperturbed guide solution whose perturbation we seek. Thus,

$$
J_{0}^{e}=\left(\gamma^{2}-\gamma_{0}^{2}\right)\left(\beta_{0}\right)^{-1 / 2},
$$

and utilizing Eqs. (16) and (18), it follows that

$$
\left(J_{0}^{e}\right)^{a}\left(\beta_{0}\right)^{-1 / 2} \Lambda_{0}^{2}=\omega^{2} \mu_{0} D_{0}^{e},
$$

where

$$
\begin{aligned}
D_{0}^{e}=\int \mathbf{E}^{a} \cdot \mathbf{\varepsilon}^{\prime} \cdot \mathbf{E} d S+\frac{1}{\mu_{0}} \int \mathbf{H}^{a} \cdot \mathbf{u}^{\prime} \cdot \mathbf{u}^{\prime} \cdot \mathbf{H} d S & +\int \mathbf{H}^{a} \cdot \mathbf{u}^{\prime} \cdot \mathbf{H} d S \\
& -\frac{1}{\omega^{2} \mu_{0}} \sum_{n=1}^{\infty} \frac{\beta_{n} \Lambda_{n}^{2} J_{n}^{e}\left(J_{n}^{e}\right)^{a}}{\left(\gamma^{2}-\gamma_{n}^{2}\right)} \\
& +\frac{\omega^{2} \mu_{0}}{k_{0}^{2}} \sum_{n=0}^{\infty} \frac{\left[\int \mathbf{f}_{n}^{a} \cdot \mathbf{\varepsilon}^{\prime} \cdot \mathbf{E} d S\right]\left[\int \mathbf{E}^{a} \cdot \mathbf{\varepsilon}^{\prime} \cdot \mathbf{f}_{n} d S\right] .}{\Omega_{n}^{2}}
\end{aligned}
$$

We can now set up a variational principle from which integral equations (16), (18), (19), and (20) are derivable. Combining Eqs. (22) and (23) we obtain the expression

$$
\frac{\left(\gamma^{2}-\gamma_{0}^{2}\right) \omega^{2} \mu_{0}}{\Lambda_{0}^{2}}=\frac{J_{0}^{e}\left(J_{0}^{e}\right)^{a}}{D_{0}^{e}} .
$$

It is readily shown that the first order variation of the right hand side of Eq. (25) with respect to the fields, subject to the condition

$$
J_{0}^{e}=\left(J_{0}^{e}\right)^{a}=D_{0}^{e} \omega^{2} \mu_{0}\left(\beta_{0}\right)^{1 / 2} \Lambda_{0}^{-2}
$$

yields the integral equations for the electromagnetic fields within the rod. Since expression (25) is amplitude independent, this condition will be automatically satisfied.

Quite analogous to the foregoing we can obtain another variational principle yielding the integral equations one would obtain starting with Eq. (5). These integral equations 
are

$$
\begin{gathered}
\mathbf{H}\left(\mathbf{x}^{\prime}\right)=\sum_{n} \frac{J_{n}^{m} \mathbf{H}_{n}\left(\mathbf{x}^{\prime}\right)}{\left(\gamma^{2}-\gamma_{n}^{2}\right)}-\frac{\omega^{2} \epsilon_{0}}{k_{0}^{2}} \sum_{n} \frac{\left[\int \mathbf{g}_{n}^{a} \cdot \mathbf{u}^{\prime} \cdot \mathbf{H} d S\right]}{\eta_{n}^{2}} \mathbf{g}_{n}\left(\mathbf{x}^{\prime}\right), \\
\frac{\boldsymbol{\varepsilon}^{\prime} \cdot \mathbf{E}\left(\mathbf{x}^{\prime}\right)}{\epsilon_{0}}=\sum_{n} \frac{\left(\alpha_{n} \beta_{n}\right)^{1 / 2} J_{n}^{m} \mathbf{E}_{n}\left(\mathbf{x}^{\prime}\right)}{\left(\gamma^{2}-\gamma_{n}^{2}\right)}-\mathbf{E}\left(\mathbf{x}^{\prime}\right),
\end{gathered}
$$

and their adjoints.

$$
\begin{aligned}
J_{n}^{m} & =\frac{\omega^{2} \epsilon_{0}}{\alpha_{n} \lambda_{n}^{2}}\left[\int \mathbf{H}_{n}^{a} \cdot u^{\prime} \cdot \mathbf{H} d S+\left(\alpha_{n} \beta_{n}\right)^{1 / 2} \int \mathbf{E}_{n}^{a} \cdot \varepsilon^{\prime} \cdot \mathbf{E} d S\right] \\
& =\left(\gamma^{2}-\gamma_{n}^{2}\right) \int \mathbf{H}_{n}^{a} \cdot \mathbf{H} d S / \alpha_{n} \lambda_{n}^{2} .
\end{aligned}
$$

Restricting ourselves to solutions which satisfy the condition $\int \mathbf{H}_{0}^{a} \cdot \mathbf{H} d S=\left(\alpha_{0}\right)^{1 / 2} \lambda_{0}^{2}$, we find

and

$$
J_{0}^{m}=\left(\gamma^{2}-\gamma_{0}^{2}\right)\left(\alpha_{0}\right)^{-1 / 2}
$$

$$
\left(J_{0}^{m}\right)^{a}\left(\alpha_{0}\right)^{-1 / 2} \lambda_{0}^{2}=\omega^{2} \epsilon_{0} D_{0}^{m}
$$

where

$$
\begin{aligned}
D_{0}^{m}=\frac{1}{\epsilon_{0}} \int \mathbf{E}^{a} \cdot \boldsymbol{\varepsilon}^{\prime} \cdot \boldsymbol{\varepsilon}^{\prime} \cdot \mathbf{E} d S+\int \mathbf{E}^{a} \cdot \boldsymbol{\varepsilon}^{\prime} \cdot \mathbf{E} d S & +\int \mathbf{H}^{a} \cdot \mathbf{u}^{\prime} \cdot \mathbf{H} d S \\
& -\frac{1}{\omega^{2} \epsilon_{0}} \sum_{n=1}^{\infty} \frac{\alpha_{n} \lambda_{n}^{2} J_{n}^{m}\left(J_{n}^{m}\right)^{a}}{\left(\gamma^{2}-\gamma_{n}^{2}\right)} \\
& +\frac{\omega^{2} \epsilon_{0}}{k_{0}^{2}} \sum_{n=0} \frac{\left[\int \mathbf{g}_{n}^{a} \cdot \mathbf{u}^{\prime} \cdot \mathbf{H} d S\right]\left[\int \mathbf{H}^{a} \cdot \mathbf{u}^{\prime} \cdot \mathbf{g}_{n} d S\right]}{\eta_{n}^{2}} .
\end{aligned}
$$

The variational expression from which these integral equations for the fields within the rod are derivable is

$$
\frac{\left(\gamma^{2}-\gamma_{0}^{2}\right) \omega^{2} \epsilon_{0}}{\lambda_{0}^{2}}=\frac{J_{0}^{m}\left(J_{0}^{m}\right)^{a}}{D_{0}^{m}}
$$

IV. Application of the variational principle. In the present application of the variational principle, we are interested in demonstrating its usefulness in improving the results obtained by perturbation methods. Consider the problem of a rectangular wave-

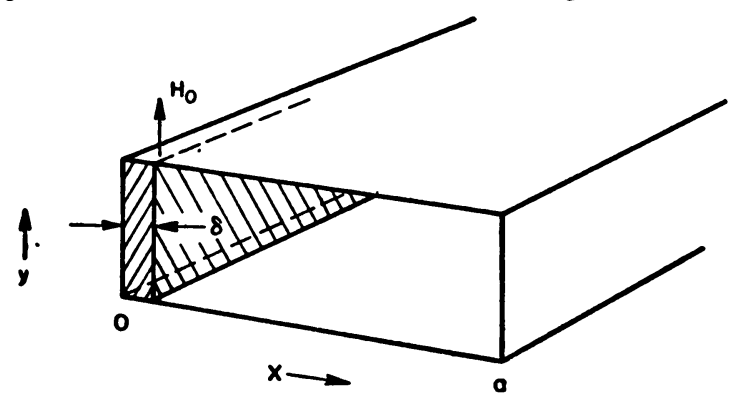

FIG. 1. A single ferrite slab in a rectangular waveguide. 
guide containing a thin ferrite slab, transversely magnetized and placed against one of the sides of the guide (Fig. 1). The exact solution of the problem [7] leads to a complicated transcendental equation for the evaluation of the propagation constant. We shall obtain the first order perturbation of the propagation constant of the lowest propagating mode of the guide, the $\mathrm{TE}_{10}$ mode, for which

$$
\left(F_{0}\right)=\left(\begin{array}{c}
0 \\
-\frac{j \omega \mu_{0}}{\pi / L} \sin \frac{\pi x}{L} \\
0
\end{array}\right) \quad \text { and } \quad\left(\alpha_{0} \mathbf{G}_{0}\right)=\left(\begin{array}{c}
-\frac{j \gamma}{\pi / L} \sin \frac{\pi x}{L} \\
0 \\
\cos \frac{\pi x}{L}
\end{array}\right) \text {. }
$$

The transversely magnetized ferrite slab has a tensor magnetic permeability

$$
(u)=\mu_{0}\left(\begin{array}{ccc}
\mu_{1} & 0 & j \kappa \\
0 & 1 & 0 \\
-j \kappa & 0 & \mu_{1}
\end{array}\right),
$$

where $\mu_{1}$ and $\kappa$ are functions of the frequency and the external DC magnetic field [8]. The electric permittivity, $\epsilon$, is a scalar, and $\mathbf{E}$ therefore divergenceless.

For first order results, the choice

$$
\mathbf{E}_{\text {trial }}=A \mathbf{F}_{0},
$$

where $A$ is a constant to be determined, is as good as any other. Its use in the perturbation formula

$$
\left(\gamma^{2}-\gamma_{0}^{2}\right) \frac{\Lambda_{0}^{2}}{\omega^{2} \mu_{0}}=\int \mathbf{F}_{0}^{a} \cdot \varepsilon^{\prime} \cdot \mathbf{E} d x+\int \alpha_{0} \mathbf{G}_{0}^{a} \cdot \mathbf{u}^{\prime} \cdot \mathbf{H} d x=j_{0}^{\mathbf{0}}
$$

which can be obtained from Eqs. (17) and (22), and is equivalent to perturbation formula (7) of [4], leads to the result

$$
\begin{aligned}
\frac{\left(\gamma^{2}-\gamma_{0}^{2}\right) \Lambda_{0}^{2}}{\omega^{2} \mu_{0}}=A\left\{\frac{\mu_{0}^{2} \epsilon^{\prime}}{(\pi / L)^{2}} d_{11}+\frac{\mu_{0}}{\omega^{2}}\left[\left(\frac{\mu_{1}^{2}-\mu_{1}-\kappa^{2}}{\mu_{1}^{2}-\kappa^{2}}\right) d_{22}\right]\right. & \\
& \left.-\frac{\mu_{0}}{\omega^{2}}\left[\frac{2 \kappa}{\left(\mu_{1}^{2}-\kappa^{2}\right)} \frac{\gamma}{\pi / L} d_{12}\right]\right\},
\end{aligned}
$$

where

$$
\begin{array}{ll}
d_{11}=\int_{0}^{\delta} \sin ^{2} \frac{\pi x}{L} d x, & d_{12}=\int_{0}^{\delta} \sin \frac{\pi x}{L} \cos \frac{\pi x}{L} d x, \\
d_{22}=\int_{0}^{\delta} \cos ^{2} \frac{\pi x}{L} d x, \text { and } & \Lambda_{0}^{2}=\frac{\omega^{2} \mu_{0}}{(\pi / L)^{2}} \frac{L}{2} .
\end{array}
$$

We have set

$$
\mathbf{H}_{\mathrm{tri \textrm {al }}}=\frac{\mu_{1}^{2}-\mu_{1}-\kappa^{2}}{\mu_{1}^{2}-\kappa^{2}} \mu_{0} \alpha_{0} \mathbf{G}_{0}-\frac{j \mu_{0}}{\mu_{1}^{2}-\kappa^{2}}\left(\mathbf{j} \times \mathbf{G}_{0}\right) \alpha_{0}
$$

and have kept only those terms which yield terms of the second order in $\delta$ or less, except 
for large values of $\epsilon$, where in the expressions containing $\epsilon$ we have kept terms yielding terms of the third order in $\delta$ or less. We have also neglected the integrations with respect to the $y$ coordinate as they all yield the same value or zero.

We find the perturbation result to be dependent on the amplitude of the trial field. In this problem for which an exact solution exists, one is able to obtain an estimate of the amplitude. In general, however, we are not as fortunate, and in such cases Eq. (23) may be used to obtain a first order approximation of the amplitude. Setting $\mathbf{E}=A \mathbf{e}$ and $\mathbf{H}=A \mathbf{h}$ we find from Eq. (23) that

$$
A=\frac{\left(j_{0}^{e}\right)^{a} \Lambda_{0}^{2}}{\omega^{2} \mu_{0} \mathfrak{D}_{0}^{e}}
$$

where

$$
\left(j_{0}^{e}\right)^{a}=\int \mathbf{e}^{a} \cdot \varepsilon^{\prime} \cdot \mathbf{F}_{0} d S+\int \mathbf{h}^{a} \cdot \boldsymbol{u}^{\prime} \cdot\left(\alpha_{0} \mathbf{G}_{0}\right) d S
$$

and

$$
A^{2} \mathfrak{T}_{0}^{e}=D_{0}^{e} .
$$

Combination of Eqs. (33) and (35) yields the variational expression

$$
\frac{\gamma^{2}-\gamma_{0}^{2}}{\omega^{2} \mu_{0}} \Lambda_{0}^{2}=\frac{j_{0}^{\circ}\left(j_{0}^{0}\right)^{a}}{\mathfrak{D}_{0}^{e}} \text {. }
$$

Before evaluating $\mathfrak{D}_{0}^{e}$ let us note that since the fields are independent of the $y$ coordinate, we can find a closed dyadic Green's function satisfying the differential equation

$$
\mathbf{d}^{a} \times \mathbf{d}^{a} \times \mathbf{N}\left(x \mid x^{\prime}\right)-k_{0}^{2} \mathbf{N}\left(x \mid x^{\prime}\right)=\mathbf{I} \delta\left(x-x^{\prime}\right),
$$

where

$$
\mathbf{d}^{a}=\frac{\partial}{\partial x} \mathbf{i}-j \gamma \mathbf{k} .
$$

Such a dyadic Green's function is given by

$$
\begin{gathered}
\mathbf{N}\left(x \mid x^{\prime}\right)=\mathbf{N}_{1}\left(x \mid x^{\prime}\right)+\mathbf{N}_{2}\left(x \mid x^{\prime}\right)-\frac{1}{k_{0}^{2}}\left(\mathbf{d}^{a} \mathbf{d}^{\prime} \cdot \mathbf{N}_{1}-\mathbf{d}^{a} \mathbf{d}^{\prime} \cdot \mathbf{N}_{2}\right), \\
\mathbf{N}_{1}\left(x \mid x^{\prime}\right)=I_{\mathfrak{N}_{1}}\left(x \mid x^{\prime}\right)
\end{gathered}
$$

where the scalar Green's function

$$
\mathfrak{N}_{1}\left(x \mid x^{\prime}\right)=-\frac{1}{2 k \sin k L} \begin{cases}\cos k\left(x-x^{\prime}-L\right) & x>x^{\prime} \\ \cos k\left(x-x^{\prime}+L\right) & x<x^{\prime}\end{cases}
$$

and satisfies the differential equation

$$
\begin{gathered}
\frac{d^{2}}{d x^{2}} \mathfrak{R}_{1}+k^{2} \mathfrak{R}_{1}=-\delta\left(x-x^{\prime}\right) . \\
\mathbf{N}_{2}\left(x \mid x^{\prime}\right)=-\mathbf{i} \mathbf{i} \mathfrak{R}_{2}+\mathbf{j} \mathbf{j} \mathfrak{R}_{2}+\mathbf{k k} \mathfrak{R}_{2}
\end{gathered}
$$

where

$$
\mathfrak{P}_{2}\left(x \mid x^{\prime}\right)=\frac{1}{2 k \sin k L} \cos k\left(x+x^{\prime}-L\right)
$$


and

$$
k^{2}=k_{0}^{2}-\gamma^{2} .
$$

In terms of this dyadic Green's function

$$
\begin{aligned}
\mathfrak{D}_{0}^{:}=\int \mathbf{e}^{a} \cdot \mathbf{\varepsilon}^{\prime} \cdot \mathbf{e} d x+\int \mathbf{h}^{a} \cdot \mathbf{u}^{\prime} \cdot \mathbf{h} d x & +\frac{1}{\mu_{0}} \int \mathbf{h}^{a} \cdot \mathbf{u}^{\prime} \cdot \mathbf{u}^{\prime} \cdot \mathbf{h} d x \\
& -\iint \mathbf{e}^{a} \cdot \mathbf{\varepsilon}^{\prime} \cdot \mathbf{N}^{T}\left(x \mid x^{\prime}\right) \cdot \mathbf{\varepsilon}^{\prime} \cdot \mathbf{e} d x d x^{\prime} \\
& -\frac{1}{\mu_{0}} \iint\left(\mathbf{h}^{a} \cdot \mathbf{u}^{\prime}\right) \cdot \mathbf{d}^{\prime} \times\left[\mathbf{d}^{a} \times \mathbf{N}\left(x \mid x^{\prime}\right)\right]^{T} \cdot\left(\boldsymbol{u}^{\prime} \cdot \mathbf{h}\right) d x d x^{\prime} \\
& +j \omega \iint\left(\mathbf{e}^{a} \cdot \mathbf{\varepsilon}^{\prime}\right) \cdot\left[\mathbf{d}^{a} \times \mathbf{N}\left(x \mid x^{\prime}\right)\right]^{T} \cdot\left(\mathbf{u}^{\prime} \cdot \mathbf{h}\right) d x d x^{\prime} \\
& -j \omega \iint\left(\mathbf{h}^{a} \cdot \mathbf{u}^{\prime}\right) \cdot\left[\mathbf{d} \times \mathbf{N}^{T}\left(x \mid x^{\prime}\right)\right] \cdot\left(\mathbf{\varepsilon}^{\prime} \cdot \mathbf{e}\right) d x d x \\
& +\frac{\omega^{2} \mu_{0}}{\left(\gamma^{2}-\gamma_{0}^{2}\right) \Lambda_{0}^{2}}\left[j_{0}^{e}\left(j_{0}^{e}\right)^{a}\right] .
\end{aligned}
$$

Inserting the trial fields, we find that to first order

$$
\mathfrak{D}_{0}^{e}=\frac{\mu_{1}}{\mu_{1}^{2}-\kappa^{2}}\left(j_{0}^{e}\right)^{a}
$$

or

$$
A=\frac{\mu_{1}^{2}-\kappa^{2}}{\mu_{1}}
$$

Thus

$$
\left(\gamma^{2}-\gamma_{0}^{2}\right) \frac{L}{2}=\omega^{2} \mu_{0} \epsilon^{\prime}\left(\frac{\mu_{1}-\kappa^{2}}{\mu_{1}}\right) d_{11}+\frac{\left(\mu_{1}^{2}-\mu_{1}-\kappa^{2}\right)}{\mu_{1}}\left(\frac{\pi}{L}\right)^{2} d_{22}-\frac{2 \kappa}{\mu_{1}}\left(\frac{\pi}{L}\right) \gamma d_{12} .
$$

It might be of interest to note that the amplitude one finds utilizing Eq. (35) is the limit of the exact amplitude as $\delta$ becomes very small.

In Fig. 2 we plot $\gamma$ for a wave at 9000 mcps as a function of $\delta$, as computed from the above equation, for a ferrite slab having a magnetization of 3000 gauss placed in an external DC magnetic field yielding an internal value of 1000 oersteds, and compare the approximate results with the exact calculation of Lax and Button [7].

While the full use of the variational method consists of the substitution of trial functions with unknown variational parameters and finding the values of these parameters which make the variational expression an extremal, we see that we may at times obtain good first order results by simply substituting completely determined trial functions. In such cases the variational expression will yield better results than the perturbation formula with the same trial field.

Acknowledgment. The author would like to express his appreciation to his colleagues for their stimulation and helpful discussions. Specifically he is indebted to Drs. L. Gold, G. S. Heller, B. Lax, H. J. Zeiger and Mr. K. J. Button. The numerical work was done by Miss Clare M. Glennon. 


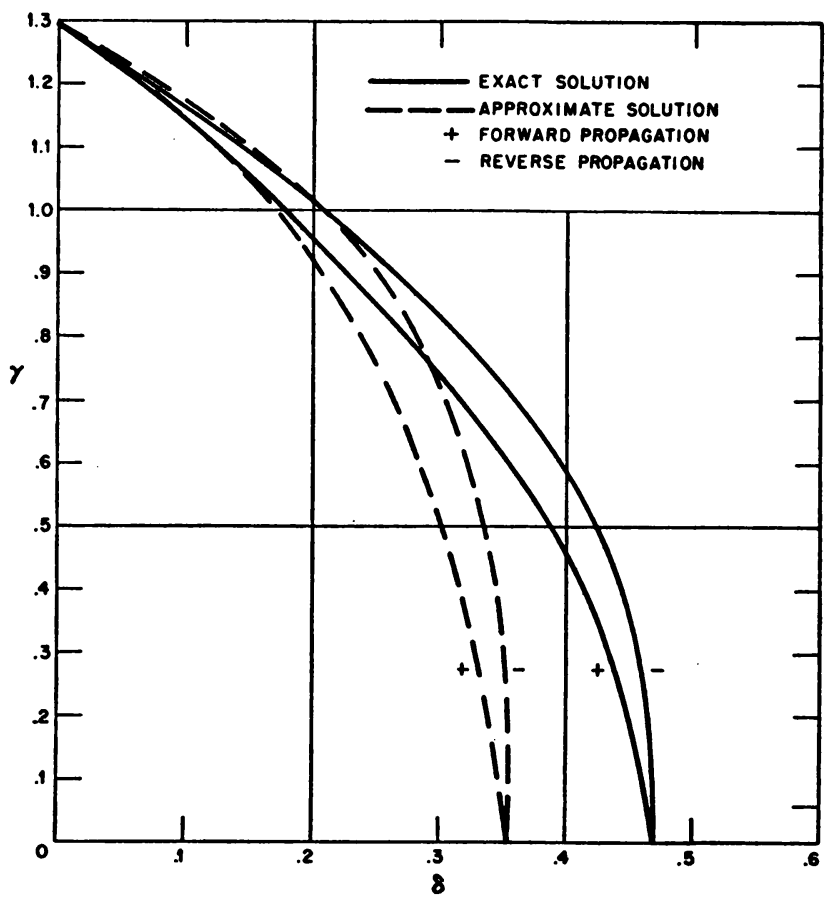

Fra. 2. Propagation constant for the $\mathrm{TE}_{1} 0$ guide mode perturbed by a single ferrite slab at 9000 mops as a function of slab thickness.

\section{REFERENCES}

1. B. Lax and G. S. Heller, private communication. Suhl and Walker, Topics in guided wave propagation through gyromagnetic media Part III, Bell System Tech. J. XXXII, II33 (1954)

2. J. Schwinger, The theory of obstacles in resonant cavities and waveguides, M.I.T., Rad. Lab. Rept. 43-34 (May 1943)

3. A. D. Berk, Variational principles for electromagnetic resonators and waveguides, I.R.E., AP 4, 104 (1956)

4. B. Lax, Frequency and loss characteristics of microwave ferrite devices, Proc. I. R. E. 44, 1368 (1956)

5. B. D. H. Tellegen, The synthesis of passive resistanceless four-poles that may violate the reciprocity relation, Phillips Research Repts. 3, 321 (1948)

6. W. Hauser, Variational principles for guided electromagnetic waves in anisotropic materials, M.I.T., Lincoln Lab. Rept. M35-61 (August 1956); not generally available

7. B. Lax and K. J. Button, Theory of new ferrite modes in rectangular waveguide, J. Appl. Phys. 26, $1184(1955)$

8. D. Polder, Phil. Mag. 40, 99 (1949) 